\title{
Vacuum Energy and Closed Orbits in Quantum Graphs
}

\author{
S. A. Fulling and J. H. Wilson
}

\begin{abstract}
The vacuum (Casimir) energy of a quantized scalar field in a given geometrical situation is a certain moment of the eigenvalue density of an associated self-adjoint differential operator. For various classes of quantum graphs it has been calculated by several methods: (1) Direct calculation from the explicitly known spectrum is feasible only in simple cases. (2) Analysis of the secular equation determining the spectrum, as in the Kottos-Smilansky derivation of the trace formula, yields a sum over periodic orbits in the graph. (3) Construction of an associated integral kernel by the method of images yields a sum over closed (not necessarily periodic) orbits. We show that for the Kirchhoff and other scale-invariant boundary conditions the sum over nonperiodic orbits in fact makes no contribution to the total energy, whereas for more general (frequency-dependent) vertex scattering matrices it can make a nonvanishing contribution, which, however, is localized near vertices and hence can be "indexed" a posteriori by truly periodic orbits. For the scale-invariant cases complete calculations have been done by both methods (2) and (3), with identical results. Indeed, applying the image method to the resolvent kernel provides an alternative derivation of the trace formula.
\end{abstract}

\section{Overview and acknowledgments}

Vacuum energy $[\mathbf{1 5}, \mathbf{1 4}, \mathbf{3 8}, \mathbf{3 7}, \mathbf{1 3}]$ is a topic in physics (quantum field theory) that has turned out to have some resonance in mathematics. It provides an application of, and a new window on, the spectral theory of second-order differential operators. In particular, there are connections with the lore surrounding the relations between the operator's spectrum and the trajectories of an associated classical-mechanical system. Insofar as energy, pressure, etc. can be localized in space, the study of vacuum energy promotes attention to localized spectral information, a somewhat neglected topic. The present article is complementary to [21]; both are directed primarily to a mathematical audience.

Much of the article is based on the undergraduate research [43] of J. Wilson under the direction of S. Fulling and G. Berkolaiko. Some results of that research have been published in $[\mathbf{9}, \mathbf{2 2}, \mathbf{2 3}]$, to which we refer for details. We are happy to acknowledge strong interactions with, and assistance from, the Texas A\&M quantum graph research group (Gregory Berkolaiko, Jonathan Harrison, Peter Kuchment, Melanie Pivarski, and Brian Winn) and Lev Kaplan of Tulane University.

2000 Mathematics Subject Classification. Primary 34B45; Secondary 34B27, 58J50, 81Q20. 
The conclusions of Sec. 6 are heavily influenced by work with graduate students Joel Bondurant [12] and Zhonghai Liu [34]. S.A.F. thanks Martin Sieber for an enlightening discussion of the special classes of paths in [42].

The research has received support from the National Science Foundation under Grants Nos. PHY-0554849 and DMS-0648786. During Spring 2007 we benefited from the hospitality of the program Analysis on Graphs and Their Applications at the Isaac Newton Institute for Mathematical Sciences at Cambridge University; both of us gave talks there that are reflected in this paper. S.A.F.'s extended stay there was made possible by a Faculty Development Leave from Texas A\&M University.

Independent research on vacuum energy in quantum graphs has been reported from Pisa $[\mathbf{7}, \mathbf{8}, \mathbf{6}]$ and Tashkent $[\mathbf{3 5}]$. The papers of the former group include deeper discussions of quantum field theories on graphs and possible applications to condensed-matter physics.

After a quick overview of the physics, we define vacuum energy mathematically in Sec. 2, giving equivalent formulas in terms of spectral density and in terms of an integral kernel. After reviewing notation for quantum graphs (Sec. 3) we demonstrate the method of multiple reflection (images) for the shortest paths in a star graph in Sec. 4. The full formula for a general graph (with scale-invariant boundary conditions) is stated in Sec. 5, and the alternative derivation from the trace formula for the spectral density is also summarized there. In Sec. 6 the more difficult situation without scale invariance (i.e., with frequency-dependent scattering amplitudes) is discussed, with a view to resolving a superficial contradiction between the image and spectral methods; as pointed out independently by J. Bolte, the trace formula for the density of states in that case contains some extra terms that have sometimes been overlooked.

\section{Vacuum energy}

2.1. Physical background. Although vacuum energy can be posed as a purely mathematical problem of intrinsic mathematical interest [21], readers with some knowledge of quantum theory will want to know something of its physical origin and significance. Unfortunately, an adequate treatment would require inserting a graduate course in quantum field theory. Here we provide a very streamlined account, concentrating on a scalar field in one spatial dimension instead of the case of greatest experimental relevance, the electromagnetic field in dimension 3. (General dimensions and general fields are potentially of interest in investigating, for instance, the implications of string theory for cosmology.) We refer to $[\mathbf{3 8}, \mathbf{3 7}, \mathbf{1 3}]$ for more information.

Consider a field $u$ satisfying the wave equation

$$
\frac{\partial^{2} u}{\partial t^{2}}=\frac{\partial^{2} u}{\partial x^{2}}
$$

on a finite interval, $0<x<L$ (for $-\infty<t<\infty$ ), and regard it as a quantum object. That is, $u(t, x)$ will become an operator (technically, an operator-valued distribution) satisfying (1) in the Heisenberg picture and satisfying certain canonical commutation relations with its time derivative. The quickest way to relate this system to the more familiar elementary quantum mechanics is to pass to Fourier 
components by

$$
u(0, x)=\sqrt{\frac{2}{L}} \sum_{k=1}^{\infty} q_{k} \sin \frac{k \pi x}{L}, \quad \frac{\partial u}{\partial t}(0, x)=\sqrt{\frac{2}{L}} \sum_{k=1}^{\infty} p_{k} \sin \frac{k \pi x}{L} .
$$

Note that $p_{k}=\frac{d q_{k}}{d t}$ and, because of (1),

$$
\frac{d^{2} q_{k}}{d t^{2}}=-\omega_{k}^{2} q_{k}, \quad \omega_{k} \equiv \frac{k \pi}{L} .
$$

We also postulate the standard relation

$$
\left[q_{k}, p_{j}\right] \equiv q_{k} p_{j}-p_{j} q_{k}=i \delta_{k j}
$$

In short, each normal mode of the field satisfies the equations defining a harmonic oscillator in quantum mechanics.

The quantum solution of the oscillator problem is well known ([36, Ch. 12], [19, Ch. 1]). The Hilbert space of a single oscillator is the closed span of vectors $\{|n\rangle\}$, $n=0, \ldots, \infty$. The energy of the vector $|n\rangle$ is $\left(\frac{1}{2}+n\right) \omega$. The Hilbert space of the entire system is now some suitably defined tensor product of these spaces. In the elementary quantum theory of free fields it is assumed that the only basis vectors that are physically realizable are those for which the occupation numbers $n_{k}$ are 0 for all but finitely many $k$. Formally, the energy of such a state, $\bigotimes_{k=1}^{\infty}\left|n_{k}\right\rangle$, is

$$
\begin{aligned}
E & =\sum_{k=1}^{\infty}\left(\frac{1}{2}+n_{k}\right) \omega_{k} \\
& =\frac{1}{2} \sum_{k=1}^{\infty} \omega_{k}+\sum_{k=1}^{\infty} n_{k} \omega_{k} .
\end{aligned}
$$

The first term in (5) is divergent, but it is independent of the state considered and is usually ignored with the justification that the zero point of energy for each oscillator is arbitrary. Under the stated assumption on $n_{k}$ the second term is finite, and the Hilbert-space closure of this set of vectors (Fock space) supports this "renormalized" energy as a densely defined self-adjoint operator.

Things become interesting when one tries to compare the energies of the vacuum states (where all $n_{k}$ are 0) for two different values of $L$. (See Fig. 1 for a higher-dimensional analogue.) From what was said in connection with (5) it would
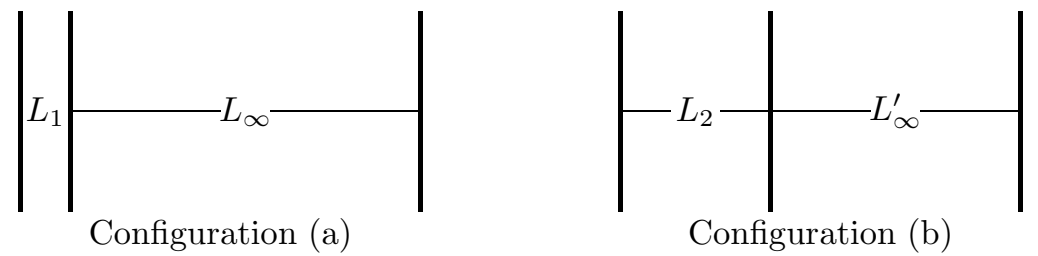

Figure 1. Two possible configurations of three parallel Dirichlet boundaries with $L_{1}<L_{2}$, idealizing electrically conducting plates. The role of the rightmost boundary in each case is merely to make it unnecessary to consider the energies of varying infinite intervals.

seem that both energies should be 0 . However, there are both theoretical and experimental reasons to believe that the two very nearby "plates" in configuration 
(a) attract each other; that is, the energy of (a) is smaller (more negative) than that of (b). The decision to replace the infinite vacuum energy $\frac{1}{2} \sum \omega_{k}$ by zero in all circumstances is too naive. The zero of the energy scale is indeed arbitrary, but the correct conclusion is now said to be that only differences of energies between different configurations are finite and meaningful. (It was also naive to expect that the vacuum of one configuration would belong to the Fock space of the other, but we need not pursue that complication here.)

The recommended calculational procedure is to modify the theory temporarily (by some cutoff procedure) to make the energy sums finite, subtract "under the summation sign", and then take a limit to remove the cutoff. It is found in many situations, notably that of Fig. 1 and all those discussed in the remainder of this paper, that the result is indeed finite and is the same for a large class of reasonable cutoff methods (regularizations). One very convenient regularization is to insert a factor $e^{-t \omega_{k}}$ in the sum (taking $t \rightarrow 0$ at the end). This method is discussed in more detail in $[\mathbf{2 1}]$. An asymptotic expansion of the sum at small $t$ (which has a close connection to both the spectral distribution of the operator in the field equation - $-\frac{\partial^{2}}{\partial x^{2}}$ in the example (1) - and the geometry of the configuration) displays divergent terms that are independent of the configuration and hence disappear when the energies are subtracted. It is common then just to ignore these terms and regard the resulting finite limit as the renormalized energy of the single configuration under study.

This line of thought breaks down when two configurations are not sufficiently similar for their energies' divergent terms to be considered identical. For example, first consider comparing one of the configurations in Fig. 1 with the situation where the middle plate is completely absent. Their energies formally differ by an infinite term associated with the missing surface. However, one may regard this surface energy as being somehow inherently associated with the material surface itself and probably rendered finite by a detailed microscopic model of the matter consituting the surface. The renormalization described earlier amounts to just excluding this surface energy from the accounting. In this way renormalized vacuum-energy calculations are considered satisfactory for studying the attraction of rigid bodies. But trouble reappears in dealing with the energy of a deformable closed surface. From a physical point of view, the dynamics of such a system much take account of the microscopic forces within the surface itself, in addition to the forces associated with the quantized field, so it is not surprising if the difference between the regularized energies of two different shapes does not converge as the cutoff is removed. With this caveat (which turns out not to be relevant to quantum graphs) we shall proceed to make a precise definition of the renormalized vacuum energy in a large class of field models and to study it as a mathematical topic.

2.2. The fundamental formulas. Let $H$ be a second-order, elliptic, selfadjoint partial differential operator, such as $-\nabla^{2}$ acting in a compact region $\Omega \subset$ $\mathbf{R}^{n}$. Assume that the spectrum of $H$ is nonnegative and discrete, and introduce the normalized eigenvectors by

$$
H \varphi_{n}=\lambda_{n} \varphi_{n}, \quad\left\|\varphi_{n}\right\|=1 .
$$

Let

$$
\omega_{n} \equiv \sqrt{\lambda_{n}} .
$$


Formally, the total vacuum energy is

$$
E \equiv \sum_{n=1}^{\infty} \frac{1}{2} \omega_{n}
$$

There are two ways to define the renormalized energy:

(1) From the density of states:

$$
E=\int_{0}^{\infty} \frac{\omega}{2}\left[\rho(\omega)-\rho_{\text {Weyl }}(\omega)\right] d \omega .
$$

Here $\rho(\omega) d \omega$ is a sum of point measures at the eigenfrequencies $\omega_{n}$, and $\rho_{\text {Weyl }}(\omega)$ stands for enough terms of the well known continuum approximation to render the integral convergent at infinity; in some cases this maneuver may introduce a new divergence at 0 and a resulting ambiguity in $E$, but that complication is beyond the scope of this paper.

(2) From the cylinder (Poisson) kernel:

$$
\begin{aligned}
T(t, x, y) & \equiv \sum_{n=1}^{\infty} \varphi_{n}(x) \overline{\varphi_{n}(y)} e^{-t \omega_{n}} \\
& =\text { integral kernel of } e^{-t \sqrt{H}} .
\end{aligned}
$$

$T$ solves a certain elliptic boundary value problem for the equation

$$
\frac{\partial^{2} T}{\partial t^{2}}=H T
$$

in $\mathbf{R}^{+} \times \Omega$. (In this context $t$ is not a time, though it can be thought of as an analytical continuation of the time variable in the original field equation, $-\frac{\partial^{2} u}{\partial t^{2}}=H u$.) Define a regularized total energy (for $t>0$ ) by

$$
\begin{aligned}
E(t) & \equiv-\frac{1}{2} \frac{\partial}{\partial t} \int_{\Omega} T(t, x, x) d x \equiv-\frac{1}{2} \frac{\partial}{\partial t} \operatorname{Tr} T \\
& =\text { Weyl terms }\left[O\left(t^{-(d+1)}\right), \text { etc. }\right]+E+O(t) .
\end{aligned}
$$

Then the renormalized total energy is defined as the limit as $t \downarrow 0$ of the regular part of $E(t)$. In other words, one examines the asymptotic behavior of $E(t)$ as $t$ approaches 0, which will normally exhibit various negative and positive powers of $t$, and extracts the constant term. The previously mentioned complication manifests itself here as a term proportional to $\ln t$, and the renormalized energy must be regarded as ambiguous modulo a term obtained by replacing $\ln t$ by an arbitrary finite constant; fortunately, the functional dependence of that term on $H$ (e.g., on the geometry of $\Omega$ ) is closely circumscribed.

In the same spirit, one defines a local vacuum energy density by forming the regularized quantity

$$
E(t, x) \equiv \frac{1}{2} \sum_{n=1}^{\infty} \omega_{n}\left|\varphi_{n}(x)\right|^{2} e^{-\omega_{n} t}=-\frac{1}{2} \frac{\partial T}{\partial t}(t, x, x)
$$

and defining the renormalized density as its regular part. 


\section{Quantum graphs}

Our intention now is to investigate vacuum energy when the region $\Omega$ is replaced by a quantum graph, $\Gamma$. We shall consider only models where $H$ is a self-adjoint extension of the Laplacian, $-\frac{d^{2}}{d x^{2}}$.

Our notation for quantum graphs $[\mathbf{3 0}, \mathbf{2 7}, \mathbf{3 1}, \mathbf{2 4}]$ is fairly standard. The graphs we consider have $V$ vertices $v$ and $B$ edges $e$ (sometimes called bonds) of lengths $L_{e}<\infty$, yielding a total length $L \equiv \sum_{e=1}^{B} L_{e}$.

To make $-\frac{d^{2}}{d x^{2}}$ self-adjoint, boundary conditions are needed. Let $H$ be such a self-adjoint extension, which acts on functions on the graph, The vacuum energy is to be found from the "cylinder equation" (11). After separation of variables in (11), we have on each edge $e$ a function $\psi_{e}$ that satisfies

$$
-\frac{d^{2}}{d x^{2}} \psi_{e}(x)=\omega^{2} \psi_{e}(x)
$$

and suitable boundary conditions at each vertex. This can equally well be done with other equations corresponding to different kernels (the heat equation, the Schrödinger equation, etc.).

The boundary conditions are implemented in terms of a vertex scattering matrix. Consider an arbitrary vertex $v$ and without loss of generality take the coordinates on all the bonds leaving $v$ to increase away from $v$. Now, consider a plane wave $e^{-i \omega x}$ on bond $e$ and incident on vertex $v$. The boundary conditions at $v$ imply a formula of the form

$$
\psi_{e^{\prime}}(x)= \begin{cases}e^{-i \omega x}+\sigma_{e e}^{(v)}(\omega) e^{i \omega x} & \text { if } e^{\prime}=e \\ \sigma_{e^{\prime} e}^{(v)}(\omega) e^{i \omega x} & \text { if } e^{\prime} \neq e\end{cases}
$$

for each $e^{\prime}$ incident on $v$. The $\sigma_{e^{\prime}}^{(v)}(\omega)$ coefficients in (15) define the vertex scattering matrix $\sigma^{(v)}(\omega)$.

A directed bond is an edge together with a choice of direction along it. Each edge $e$ corresponds to two directed bonds, say $e^{+}$and $e^{-}$, so the set of directed bonds has size $2 B$. A directed bond will be denoted by a greek letter ( $\alpha$ or $\beta$ usually). If $\alpha=e^{+}$or $\alpha=e^{-}$, we say $\alpha$ is associated with edge $e$. Also, $\bar{\alpha}$ is the reversal of $\alpha$ (i.e. if $\alpha=e^{+}$, then $\bar{\alpha}=e^{-}$).

Using directed bonds we can define a $2 B \times 2 B$ scattering matrix such that $S_{\beta \alpha}(\omega)=\sigma_{e^{\prime} e}^{(v)}(\omega)$ if $\alpha$ is associated with $e$ terminating at $v$ and $\beta$ is associated with $e^{\prime}$ originating at $v$. Otherwise, $S_{\beta \alpha}=0$. The matrix $S$ is the notation of Kottos and Smilansky; Kostrykin and Schrader have an equivalent matrix, $\mathfrak{S}(\omega)$, indexed by edge-ends instead of directed bonds. These two matrices are related by

$$
\mathfrak{S}=S J, \quad J_{\beta \alpha} \equiv \delta_{\beta \bar{\alpha}} .
$$

The most common kind of boundary condition, called Kirchhoff, natural, or sometimes Neumann, calls for continuity of $u$ and conservation of its "flux" at each vertex $v$ : If $d_{v}$ vertices meet at $v$, then

$$
u_{e}(v)=\text { same for all } e, \quad \sum_{e=1}^{d_{v}} u_{e}^{\prime}(v)=0 .
$$


As usual, the derivative in Eq. (16) is taken outward from the vertex $v$. The corresponding vertex scattering matrix is

$$
\sigma_{e e^{\prime}}^{(v)}=\frac{2}{d_{v}}-\delta_{e e^{\prime}}
$$

More generally, the boundary conditions for the Laplacian of a graph are called scale-invariant if the resulting scattering matrices are independent of $\omega$. These are precisely the conditions that do not mix the endpoint values of $u$ with those of $u^{\prime}$. A detailed discussion of equivalent conditions appears in [23]. The key lemma we need here is the following, established by Kostrykin and Schrader $[\mathbf{2 7}, \mathbf{2 8}, \mathbf{2 6}]$ :

Lemma 3.1. For any scale-invariant boundary conditions, $\mathfrak{S}^{2}=(S J)^{2}=I$. (Equivalently, for all $v,\left(\sigma^{(v)}\right)^{2}=I$.)

\section{Star graphs}

4.1. A star graph with equal edge lengths treated directly. As a simple first example $[\mathbf{2 2}]$ we consider star graphs all of whose edges have the same length (Fig. 2). Its eigenfrequencies are

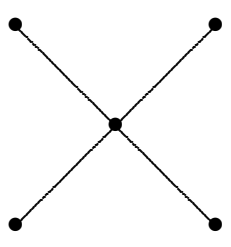

FIgURE 2. A star graph with 4 edges of length $a$.

$$
\omega= \begin{cases}\frac{n \pi}{a} & \text { with multiplicity } 1, \\ \left(n+\frac{1}{2}\right) \frac{\pi}{a} & \text { with multiplicity } B-1 .\end{cases}
$$

For the Kirchhoff boundary conditions (16) the cylinder trace can be calculated exactly:

$$
\begin{aligned}
\operatorname{Tr} T(t) & =\sum_{n=1}^{\infty} e^{-n \pi t / a}+(B-1) e^{-\pi t / 2 a} \sum_{n=0}^{\infty} e^{-n \pi t / a} \\
& =\frac{1+(B-1) e^{-\pi t / 2 a}}{1-e^{-\pi t / a}} \\
& =\frac{a}{\pi t}+\frac{1}{2}+\frac{\pi t}{12 a}+(B-1)\left[\frac{a}{\pi t}-\frac{\pi t}{24 a}\right]+\cdots \\
& =\frac{L}{\pi t}+\frac{1}{2}-\frac{(B-3) \pi t}{24 a}+O\left(t^{2}\right) .
\end{aligned}
$$

Therefore, the renormalized total energy, according to (12) and the discussion following, is

$$
E=\frac{(B-3) \pi}{48 a}
$$

Note the sign change at $B=3[\mathbf{2 2}]$; the sign of Casimir energy in various situations is far from completely understood physically. 
4.2. The general star graph treated by multiple reflection. When the edge lengths are not equal, exact spectral calculations are more difficult. However, the cylinder kernel can still be constructed exactly by an appropriate generalization of the method of images $[\mathbf{4 3}, \mathbf{9}]$. One starts with the "free" cylinder kernel on the real line,

$$
T_{0}(t, x, y)=\frac{t}{\pi} \frac{1}{t^{2}+(x-y)^{2}},
$$

and constructs the kernel on $\Gamma$ satisfying the necessary differential equation (11) and boundary conditions by adding the propagation of $T_{0}$ from $y \in \Gamma$ to $x \in \Gamma$ via all possible paths, using the scattering matrix (17) (or its analogue for any other scale-invariant model) to calculate the amplitude for reflection or transmission at each vertex. To take the trace, we are interested in $y=x$; in particular, $x$ and $y$ will lie on the same edge $e$, and the relevant piece of the kernel will be a function $T_{e}^{e}(t, x, y)$ on $\mathbf{R}^{+} \times e \times e$ (where $e$ is associated with the interval $\left(0, L_{e}\right)$ ).

This process has been derived in full generality (for scale-invariant boundary conditions) in [43]. (More general boundary conditions will be treated elsewhere.) Here, for expository purposes, we consider a star graph with Kirchhoff conditions and write out only the simplest few classes of paths and the corresponding expressions. In [43], these terms are taken from Ch. 3, Sec. C, Eq. (3.27), with slight changes in notation. Let the pertinent edge be $e=1$. We compile the path of zero length, then the two paths that visit exactly one vertex (for which conceptual "images" are shown), then the two paths that visit two vertices, the three path types that visit three, the four types that visit four, and the seven types that visit five:

$$
T_{1}^{1}(t, x, x)=T_{0}(t, x, x)
$$

$$
\begin{aligned}
& +\underset{*}{\leftarrow} \cdots \times \quad+T_{0}\left(t, x, 2 L_{1}-x\right) \\
& +\left(\frac{2}{B}-1\right) T_{0}(t, x,-x) \\
& +\left(\frac{2}{B}-1\right) T_{0}\left(t, x, x-2 L_{1}\right) \\
& + \text { reverse } \quad+\text { same with } y=x+2 L_{1}\left(\text { instead of } x-2 L_{1}\right) \\
& +\sum_{e \neq 1}\left(\frac{2}{B}\right)^{2} T_{0}\left(t, x,-2 L_{e}-x\right) \\
& +\left(\frac{2}{B}-1\right)^{2} T_{0}\left(t, x,-2 L_{1}-x\right) \\
& +\left(\frac{2}{B}-1\right) T_{0}\left(t, x, 4 L_{1}-x\right)
\end{aligned}
$$
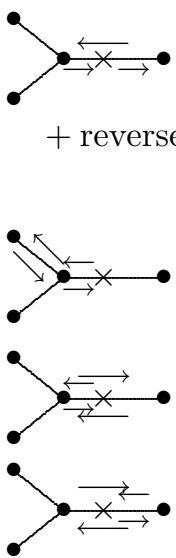


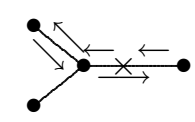

+ reverse

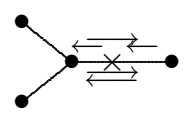

+ reverse
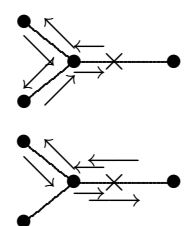

+ reverse
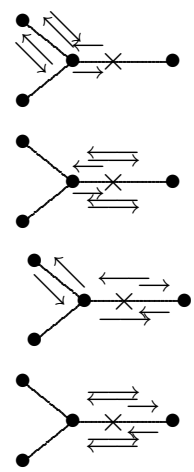

$+\sum_{e \neq 1}\left(\frac{2}{B}\right)^{2} T_{0}\left(t, x, x+2 L_{1}+2 L_{e}\right)$

+ same with $y=x-2 L_{1}-2 L_{e}$

$+\left(\frac{2}{B}-1\right)^{2} T_{0}\left(t, x, x+4 L_{1}\right)$

+ same with $y=x-4 L_{1}$

$$
\begin{aligned}
& +\sum_{1 \neq e \neq e^{\prime} \neq 1}\left(\frac{2}{B}\right)^{3} T_{0}\left(t,-2 L_{e}-2 L_{e^{\prime}}-x, x\right) \\
& +\sum_{e \neq 1}\left(\frac{2}{B}\right)^{2}\left(\frac{2}{B}-1\right) T_{0}\left(t,-2 L_{1}-2 L_{e}-x, x\right)
\end{aligned}
$$

+ same

$+\sum_{e \neq 1}\left(\frac{2}{B}\right)^{2}\left(\frac{2}{B}-1\right) T_{0}\left(t,-4 L_{e}-x, x\right)$

$+\left(\frac{2}{B}-1\right)^{3} T_{0}\left(t,-4 L_{1}-x, x\right)$

$+\sum_{e \neq 1}\left(\frac{2}{B}\right)^{2} T_{0}\left(t, 4 L_{1}+2 L_{e}-x, x\right)$

$+\left(\frac{2}{B}-1\right)^{2} T_{0}\left(t, 6 L_{1}-x, x\right)$

$+\cdots$.

The periodic paths are precisely those with an even number of vertices.

The total energy is to be obtained from the trace,

$$
\operatorname{Tr} T=\sum_{e=1}^{B} \int_{0}^{L_{e}} T_{e}^{e}(t, x, x) d x .
$$

At this point distinctions between the roles of various types of path become evident. The contributions to the trace of paths with fewer than three vertices are

$$
\begin{array}{cc}
\frac{L}{\pi t} \quad(\text { leading Weyl term) } & \text { path of zero length } \\
\cline { 2 - 2 }+\frac{1}{2 \pi} \sum_{e} \tan ^{-1}\left(\frac{2 L_{e}}{t}\right) & \begin{array}{c}
\text { retracing } \\
\text { nonperiodic path }
\end{array} \\
+\frac{1}{2 \pi}\left(\frac{2}{B}-1\right) \sum_{e} \tan ^{-1}\left(\frac{2 L_{e}}{t}\right) & \begin{array}{c}
\text { retracing } \\
\text { nonperiodic path }
\end{array} \\
+\frac{t}{\pi}\left(\frac{2}{B}-1\right) \sum_{e} \frac{L_{e}}{t^{2}+\left(2 L_{e}\right)^{2}} & 2 \text { periodic orbits } \\
+ \text { same. } &
\end{array}
$$


The paths with three vertices are retracing nonperiodic paths. The corresponding integrals in (23) collectively yield

$$
-\frac{1}{\pi B} \sum_{e} \tan ^{-1}\left(\frac{2 L_{e}}{t}\right)
$$

from their lower limits and

$$
\frac{1}{\pi B}\left[\left(\frac{2}{B}-1\right) \sum_{e} \tan ^{-1}\left(\frac{4 L_{e}}{t}\right)+\frac{2}{B} \sum_{e \neq e^{\prime}} \tan ^{-1}\left(\frac{2 L_{e}+2 L_{e^{\prime}}}{t}\right)\right]
$$

from their upper limits. Because (25) is the negative of the sum of the similar $\tan ^{-1}$ (two lengths) terms in (24), the $\tan ^{-1}$ (four lengths) terms (26) are the cumulation so far from the nonperiodic paths.

The contributions to the trace of the remaining terms in (22), rendered a bit more schematically, are

$$
\begin{array}{cc}
\frac{2 t}{\pi}\left(\frac{2}{B}\right)^{2} \sum_{e \neq e^{\prime}} \frac{L_{e}}{t^{2}+\left(2 L_{e}+2 L_{e^{\prime}}\right)^{2}} & \text { periodic orbits } \\
+\frac{2 t}{\pi}\left(\frac{2}{B}-1\right)^{2} \sum_{e} \frac{L_{e}}{t^{2}+\left(4 L_{e}\right)^{2}} & \text { periodic orbits } \\
+\sum \tan ^{-1}(\operatorname{six} \text { lengths }) & \text { nonretracing } \\
-\frac{1}{2 \pi}(B-2)\left(\frac{2}{B}\right)^{3} \sum_{e \neq e^{\prime}} \tan ^{-1}\left(\frac{2 L_{2}+2 L_{e^{\prime}}}{t}\right) & \text { nonperiodic paths } \\
+\sum \tan ^{-1}(\operatorname{six} \text { lengths }) & \\
-\frac{1}{\pi}\left(\frac{2}{B}\right)^{2}\left(\frac{2}{B}-1\right) \sum_{e \neq e^{\prime}} \tan ^{-1}\left(\frac{2 L_{2}+2 L_{e^{\prime}}}{t}\right) & \text { nonretracing } \\
+\sum \tan ^{-1}(\operatorname{six} \text { lengths }) & \text { nonperiodic paths } \\
+[\text { negative of }(26)] . & \text { retracing } \\
\text { nonperiodic paths }
\end{array}
$$

The cancellation involving the retracing nonperiodic paths hinges on the identity

$$
(B-1)\left(\frac{2}{B}\right)^{2}+\left(\frac{2}{B}-1\right)^{2}=1 .
$$

Furthermore, because

$$
(B-2) \frac{2}{B}+2\left(\frac{2}{B}-1\right)=0
$$

the $\tan ^{-1}$ (four lengths) contributions of the nonretracing nonperiodic paths add to zero!

These patterns will persist to all orders: The terms from nonretracing nonperiodic paths cancel; the terms from retracing nonperiodic paths telescope to $\frac{1}{\pi} \tan ^{-1}(\infty)=\frac{1}{2}$ (a constant!), the second (and last) term of the Weyl series; the terms from periodic paths have the form $C t+O\left(t^{3}\right)$. Only the periodic terms contribute to the total energy,

$$
E \equiv-\left.\frac{1}{2} \frac{\partial}{\partial t}\left[\operatorname{Tr} T-\frac{L}{\pi t}\right]\right|_{t=0}=-\frac{1}{2} \sum_{\text {periodic orbits }} C .
$$




\section{General scale-invariant graphs}

5.1. Treatment by multiple reflection. The construction applied to Kirchhoff star graphs in the previous section extends to a systematic sum over paths of all lengths, in a general graph, and for general scale-invariant boundary conditions. We define

$$
\begin{gathered}
u(z) \equiv T_{0}(t, z, 0)=\frac{t}{\pi} \frac{1}{t^{2}+z^{2}}, \quad u_{e}(x) \equiv T_{e}^{e}(t, x, x), \\
A_{\mathbf{q}} \equiv S_{\alpha_{n}, \alpha_{n-1}} \cdots S_{\alpha_{2}, \alpha_{1}} \\
\text { for path } \mathbf{q},
\end{gathered}
$$

and the key formula $[\mathbf{4 3}]$ is

$$
\begin{aligned}
u_{e}(x)= & u(0) \\
& +\sum_{\mathbf{p}}\left[A_{\bar{\alpha} \mathbf{p} \alpha} u\left(L_{\mathbf{p}}+2 L_{e}-2 x\right)+A_{\alpha \mathbf{p} \bar{\alpha}} u\left(-L_{\mathbf{p}}-2 x\right)\right. \\
& \left.+A_{\alpha \mathbf{p} \alpha} u\left(-L_{\mathbf{p}}-L_{e}\right)+A_{\bar{\alpha} \mathbf{p} \bar{\alpha}} u\left(-L_{\mathbf{p}}+L_{e}\right)\right] \\
= & \text { direct }+ \text { nonperiodic }+ \text { periodic. }
\end{aligned}
$$

The lemma of Kostrykin and Schrader stated above plays a major role here; its consequences are that nonretracing nonperiodic paths cancel, and retracing nonperiodic paths sum to a constant. Additionally, the fact that we use scale-invariant scattering matrices allowed us to write the function $u$ times an amplitude $A_{\mathbf{q}}$ in Eq. (31).

The method applied here directly to the cylinder kernel is essentially the same as that applied by Roth [40] to the heat kernel (for the Kirchhoff case only), and in [5] and [29] to the resolvent kernel (for the general case). The result of [29] was used in $[\mathbf{2 6}]$ to derive the heat kernel and its trace.

5.2. Treatment by the trace formula. For scale-invariant graphs, the Kottos-Smilansky [30] trace formula for the eigenvalue density is

$$
\rho(\omega)=\frac{L}{\pi}+C \delta(\omega)+\operatorname{Re} \sum_{\mathbf{p}} \sum_{r=1}^{\infty} \frac{L_{\mathbf{p}}}{\pi} A_{\mathbf{p}}^{r} e^{i \omega r L_{\mathbf{p}}} .
$$

Although periodic orbits appear in this formula, it is not derived in the first instance by tracing out classical paths, but rather by analyzing a certain secular determinant. The constant $C$ is hard to determine from the determinant; it doesn't contribute to the vacuum energy anyway. (See, however, [23].) The sum in (32) is over primitive periodic orbits and their repetitions, $r$.

From (32) one calculates the total energy

$$
E=\int_{0}^{\infty} \frac{\omega}{2} \rho_{\text {osc }}(\omega) d \omega=-\frac{1}{2 \pi} \operatorname{Re} \sum_{\mathbf{p}, r} \frac{A_{\mathbf{p}}{ }^{r}}{L_{\mathbf{p}} r^{2}}
$$

This is the same result as given by the method of multiple reflections, but the calculation is more efficient. On the other hand, the trace method does not easily yield the local energy density. Detailed proofs of (33), including discussion of convergence issues, appear in $[43,9]$. In fact, $E$ is a $C^{\infty}$ function of the edge lengths $L_{e}$ in the domain where all $L_{e}>0[\mathbf{9}]$. 
5.3. Many routes to the same goal. In summary, there is a progression of approaches that can be (and have been) taken to calculate the vacuum energy in quantum graphs:

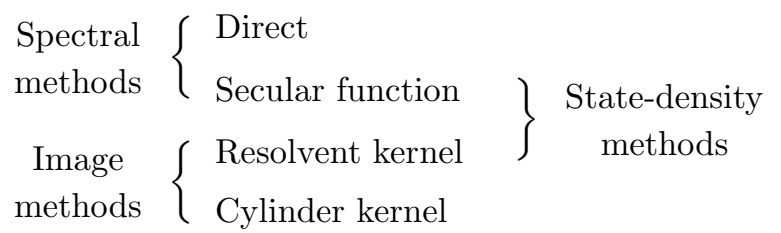

In some contexts the methods are interchangeable, but in other respects they have different virtues. (For example, if one wants to know the spectrum of the system, not just the vacuum energy, then one of the first three methods is indicated, but if one wants the energy density, the cylinder method is better.)

\section{6. "Robin" problems}

Boundary conditions that are not scale-invariant, but are nevertheless local in the sense that they relate the values of the field and its derivatives at the endpoints of edges that meet at vertices, are the graph analogue of Robin boundary conditions on intervals or billiards. Here we make some qualitative remarks upon this situation, with emphasis on how the multiple-reflection and trace-formula approaches are to be reconciled. (Similar observations have been made by Bolte and Endres $[\mathbf{1 0}, \mathbf{1 1}]$.)

The previous discussions do not apply, because two things go wrong simultaneously. First, the fact that the scattering matrix is frequency-dependent implies that the reflection law for the cylinder kernel (or the other standard time-dependent kernels, those for the heat, wave, and Schrödinger equations) is nonlocal in $t$. (This effect has been studied in detail, for a single Robin endpoint, in [12].) Second, the Kostrykin-Schrader identity $\mathfrak{S}^{2}=I$ fails, and consequently nonperiodic closed paths make nontrivial contributions to the traces of kernels (including the total vacuum energy).

These issues already arise for the simplest of compact quantum graphs, the interval, when it is equipped with a Robin boundary condition:

$$
0 \bullet-u^{\prime}(0)=\alpha u(0) \quad(\alpha>0), \quad u(L)=0 .
$$

The mathematical behavior and quantum field theory of this model have been studied in $[\mathbf{3 2}, \mathbf{3 3}, \mathbf{3 9}, \mathbf{1 2}, \mathbf{3 4}]$. (A Robin-type condition at the central vertex of an infinite star graph was treated in $[\mathbf{2 0}]$ and [7].)

It is instructive to investigate

(1) the local energy density, $E(x)$;

(2) the total energy, $E$;

(3) the density of states, $\rho(\omega)$.

(There is also a local spectral density, but we shall not belabor that point here. See [21].) In general, each Robin quantity is equal to the corresponding Neumann quantity plus a correction dependent on $\alpha$.

6.1. Analysis by multiple reflection. The following picture emerges from $[\mathbf{1 2}, \mathbf{3 4}]$ and the analogy with billiards discussed below. There are three types of closed paths (besides the one of zero length): 
(1) Gutzwiller type (periodic orbits). For them $E(x)$ is uniform in space. $E$ carries a factor of order $L$ (is an "extensive" quantity); $\rho(\omega)$ also carries a factor of order $L$ and is oscillatory.

(2) Weyl type (roughly analogous to retracing nonperiodic paths). For them $E(x)$ is concentrated near an endpoint. $E$ involves no factor $L$ in its numerator; $\rho(k)$ has no factor $L$ and is not oscillatory. These terms in $\rho$ are the boundary terms in the well-known Weyl series [16].

(3) Sieber type (roughly analogous to nonretracing nonperiodic paths, but no longer ignorable as they were in Sec. 4). For them $E(x)$ is concentrated near an endpoint. $E$ has no factor $L ; \rho(\omega)$ has no factor $L$ but is oscillatory. These terms are not part of the Weyl series, and they are of higher semiclassical or adiabatic order than Gutzwiller terms. ("Semiclassical" becomes meaningful when $\hbar$ is introduced via $\frac{1}{k L}=\frac{\hbar}{p L}$.)

To clarify this classification we point out the analogy with the well-known situation in rectangular billiards.

(1) Periodic paths yield the Gutzwiller oscillations [3, 17, 25] (Fig. 3).

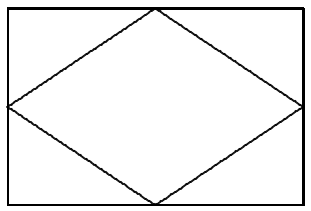

FiguRe 3. A periodic path in a rectangle.

(2) Nonperiodic paths perpendicular to edges, or hitting corners, yield the edge and corner terms in the Weyl series (Fig. 4).

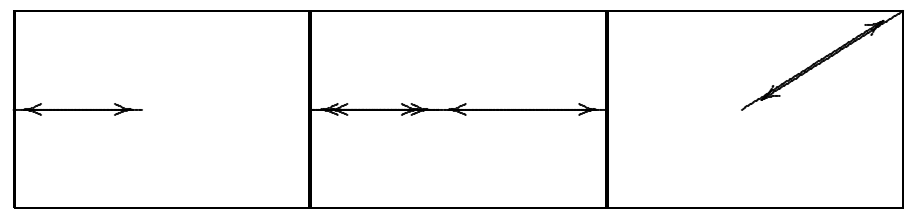

FiguRE 4. Typical retracing nonperiodic closed paths in a rectangle. The one in the middle bounces twice from the left side of the rectangle and once from the right; such "long" paths individually make nonsingular contributions to the boundary vacuum energy, but their effects must be combined with that of the single-bounce paths to constitute the entire Weyl surface term.

(3) Nonperiodic paths with transverse drift yield non-Weyl, non-Gutzwiller terms associated with the edge regarded as a special periodic orbit (Fig. 5). We call these Sieber paths because of their prominence in [42, Sec. 2.3] and $[\mathbf{4 1}$, Sec. 2.2]. (Of course, the choice of the labels "Gutzwiller" and "Sieber" is a somewhat arbitrary convenience.) 


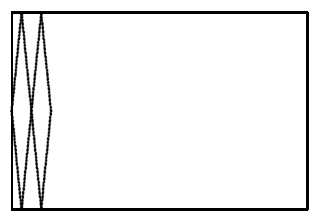

FiguRe 5. A nonperiodic orbit that contributes to a correction to $\rho$. The full effect of the family of similar orbits near the left edge of the rectangle can be described as a contribution of the edge itself, a periodic orbit [42]. The resulting contribution to the energy density is concentrated near that boundary [18].

6.2. Analysis by the trace formula. It must be possible to calculate $E$ from (9) and the trace formula (32). At first glance, however, there is a contradiction with the foregoing considerations, because the trace formula refers only to Weyl terms and periodic paths.

Let us examine the situation more closely. The counting function, $N(\omega) \equiv$ $\int \rho(\omega) d \omega$, satisfies $N(\omega)=0$ when $\omega<0$. When $\omega>0$, for the Robin-Dirichlet interval (34) one has

$$
N(\omega)=\frac{L \omega}{\pi}+C^{\prime}+\frac{1}{\pi} \tan ^{-1} \frac{\omega}{\alpha}+\operatorname{Im} \sum_{\mathbf{p}, r} \frac{1}{\pi r} A_{\mathbf{p}}^{r} e^{i \omega r L_{\mathbf{p}}},
$$

hence by differentiation

$$
\begin{aligned}
\rho(\omega)= & \frac{L}{\pi}+C \delta(\omega)+\frac{\alpha}{\pi} \frac{1}{\omega^{2}+\alpha^{2}} \\
& +\operatorname{Re} \sum_{\mathbf{p}, r} \frac{L_{\mathbf{p}}}{\pi} A_{\mathbf{p}}{ }^{r} e^{i \omega r L_{\mathbf{p}}}+\operatorname{Im} \sum_{\mathbf{p}, r} \frac{1}{\pi} A_{\mathbf{p}}^{r-1} \frac{d A_{\mathbf{p}}}{d \omega} e^{i \omega r L_{\mathbf{p}}} \\
= & \text { Weyl series }+ \text { Gutzwiller terms }+ \text { Sieber terms. }
\end{aligned}
$$

Here there is only one $\mathbf{p}$, with $L_{\mathbf{p}}=2 L$, and $-A_{\mathbf{p}}$ is the Robin reflection coefficient,

$$
\sigma_{11}^{(0)}=\frac{\omega-i \alpha}{\omega+i \alpha} ; \quad \frac{d \sigma}{d \omega}=\frac{2 i \alpha}{(\omega+i \alpha)^{2}} .
$$

So the factor

$$
A_{\mathbf{p}}^{r-1}\left(i L_{\mathbf{p}} A_{\mathbf{p}}+\frac{d A_{\mathbf{p}}}{d \omega}\right)
$$

that emerges from the Gutzwiller and Sieber terms taken together contains the factor

$$
L+\frac{\alpha}{\omega^{2}+\alpha^{2}}
$$

- the same factor that appears in (36) in the Weyl terms. The first term in (38) is extensive. The second term, which vanishes in a scale-invariant model, is associated with the boundary. The Sieber terms are smaller than the Gutzwiller terms by factors of order

$$
A_{\mathbf{p}}^{r-1} \frac{d A_{\mathbf{p}}}{d \omega} / L_{\mathbf{p}} A_{\mathbf{p}}^{r}=O\left(\frac{1}{L \sqrt{\omega^{2}+\alpha^{2}}}\right) .
$$

Thus the resolution of the apparent paradox is twofold: 
- The $\omega$ derivatives of the amplitudes $A_{\mathbf{p}}$ cannot be neglected, and they yield Sieber terms.

- In global quantities (density of states, total energy) the Sieber terms, being associated with an endpoint instead of a whole edge of the graph, are "indexed" by periodic orbits. In local quantities, though, they must be assigned to nonperiodic closed orbits.

In [12] the convergence of the closed-path sums for the Robin interval is studied numerically and analytically. The sum is conditionally distributionally convergent, even for $\omega$ on the real axis, but the strength of the $\delta(\omega)$ at the origin is sensitive to the ordering of the terms.

\section{Final comments}

The image method is the classic approach to boundary-value problems with only one essential spatial dimension. In multidimensional problems the closest analogue of the image method is multiple reflection in billiards [1] or multiple scattering in potentials $[\mathbf{2}, \mathbf{4}]$. These should not be confused with geometrical optics $[\mathbf{3}]$ or semiclassics [4] in those respective situations; in the latter, stationary-phase approximations are made. The beauty of quantum graphs (and also of rectangles and parallelepipeds) is that the

$$
\text { spectrum } \leftrightarrow \text { paths }
$$

connection can be separated from the high-frequency approximation, with calculations still feasible. (In higher dimensions, the paths in the multiple-reflection/scattering expansion are generally not "classical". In graphs, the paths are classical on the edges and nonclassical at the vertices, which are singularities where a semiclassical picture breaks down.)

Vacuum energy density (or the cylinder kernel) is more sensitive to global geometry than is the (small- $t$ asymptotics of the) heat kernel. It contains the extra information in a more accessible and intuitive form than does the resolvent kernel (or the local density of states), because magnitudes are easier to grasp than oscillations; its sign still contains some phase information. It deserves more study by mathematicians.

\section{References}

[1] R. Balian and C. Bloch, Distribution of eigenfrequencies for the wave equation in a finite domain. I, Ann. Phys. 60 (1970) 401-447.

[2] R. Balian and C. Bloch, Asymptotic evaluation of the Green's function for large quantum numbers, Ann. Phys. 63 (1971) 592-606.

[3] R. Balian and C. Bloch, Distribution of eigenfrequencies for the wave equation in a finite domain. III, Ann. Phys. 69 (1972) 76-160.

[4] R. Balian and C. Bloch Solution of the Schrödinger equation in terms of classical paths, Ann. Phys. 85 (1974) 514-545.

[5] F. Barra and P. Gaspard. Transport and dynamics on open quantum graphs, Phys. Rev. 65 (2001) 016205.

[6] B. Bellazzini, M. Burrello, M. Mintchev, and P. Sorba, Quantum field theory on star graphs, this volume.

[7] B. Bellazzini and M. Mintchev, Quantum fields on star graphs, J. Phys. A 39 (2006) 1110111117.

[8] B. Bellazzini, M. Mintchev, and P. Sorba, Bosonization and scale invariance on quantum wires, J. Phys. A 40 (2007) 2485-2508.

[9] G. Berkolaiko, J. Harrison, and J. H. Wilson, Mathematical aspects of vacuum energy on quantum graphs, preprint (arXiv:0711.2707). 
[10] J. Bolte and S. Endres, Trace formulae for quantum graphs, this volume.

[11] J. Bolte and S. Endres, The trace formula for quantum graphs with general self-adjoint boundary conditions, in preparation.

[12] J. D. Bondurant and S. A. Fulling, The Dirichlet-to-Robin transform, J. Phys. A 38 (2005) $1505-1532$.

[13] M. Bordag, U. Mohideen, and V. M. Mostepanenko, New developments in the Casimir effect, Phys. Reps. 353 (2001) 1-205.

[14] L. S. Brown and G. J. Maclay, Vacuum stress between conducting plates: An image solution, Phys. Rev. 184 (1969) 1272-1279.

[15] H. B. G. Casimir, On the attraction between two perfectly conducting plates, Konink. Nederl. Akad. Weten., Proc. Sec. Sci. 51 (1948) 793-795.

[16] C. Clark, The asymptotic distribution of eigenvalues and eigenfunctions for elliptic boundary value problems, SIAM Rev. 9 (1967) 627-646.

[17] Y. Colin de Verdière, Spectre du laplacien et longuers des géodésiques périodiques. II, Compos. Math. 27 (1973) 159-184.

[18] R. Estrada, S. A. Fulling, L. Kaplan, K. Kirsten, Z. H. Liu, and K. A. Milton, Vacuum stress-energy density and its gravitational implications, J. Phys. A, in press.

[19] S. A. Fulling, Aspects of Quantum Field Theory in Curved Space-Time, Cambridge University Press, Cambridge, 1989.

[20] S. A. Fulling, Local spectral density and vacuum energy near a quantum graph vertex, in Quantum Graphs and Their Applications (G. Berkolaiko et al., eds.), Contemp. Math., vol. 415, Amer. Math. Soc., Providence, RI, 2006, pp. 161-172.

[21] S. A. Fulling, Vacuum energy as spectral geometry, Sym. Integrab. Geom.: Meth. Appl. 3 (2007) 094.

[22] S. A. Fulling, L. Kaplan, and J. H. Wilson, Vacuum energy and repulsive Casimir forces in quantum star graphs, Phys. Rev. A 76 (2007) 012118.

[23] S. A. Fulling, P. Kuchment, and J. H. Wilson, Index theorems for quantum graphs, J. Phys. A 40 (2007) 14165-14180.

[24] S. Gnutzmann and U. Smilansky, Quantum graphs: Applications to quantum chaos and universal spectral statistics, Adv. Phys. 55 (2006) 527-625.

[25] M. C. Gutzwiller, Chaos in Classical and Quantum Mechanics, Springer, New York, 1990, and related papers.

[26] V. Kostrykin, J. Potthoff, and R. Schrader, Heat kernels on metric graphs and a trace formula, in Adventures in Mathematical Physics (F. Germinet and P. D. Hislop, eds.), Contemp. Math., vol. 447, Amer. Math. Soc., Providence, RI, 2007, pp. 175-198.

[27] V. Kostrykin and R. Schrader, Kirchhoff's rule for quantum wires, J. Phys. A 32 (1999) $595-630$.

[28] V. Kostrykin and R. Schrader, Kirchhoff's rule for quantum wires. II: The inverse problem with possible applications to quantum computers, Fortschr. Phys. 48 (2000) 703-716.

[29] V. Kostrykin and R. Schrader, Laplacians on metric graphs: Eigenvalues, resolvents and semigroups, in Quantum Graphs and Their Applications (G. Berkolaiko et al., eds.), Contemp. Math., vol. 415, Amer. Math. Soc., Providence, RI, 2006, pp. 201-225.

[30] T. Kottos and U. Smilansky, Periodic orbit theory and spectral statistics for quantum graphs, Ann. Phys. 274 (1999) 76-124.

[31] P. Kuchment, Quantum graphs. I: Some basic structures, Waves Random Media 14 (2004) S107-S128.

[32] S. L. Lebedev, Casimir effect in the presence of an elastic boundary, Zh. Eksp. Teor. Fiz. 110 (1996) 769-792 [JETP 83 423-434].

[33] S. L. Lebedev, Vacuum energy and Casimir force in the presence of a dimensional parameter in the boundary condition, Yad. Fiz. 64 (2001) 1413-1422 [Phys. At. Nuclei 64 1337-1346].

[34] Z. H. Liu and S. A. Fulling, Casimir energy with a Robin boundary: The multiple-reflection cylinder-kernel expansion, New J. Phys. 8 (2006) 234.

[35] D. U. Matrasulov, J. R. Yusupov, P. K. Khabibullaev, and A. A. Saidov, Casimir effect for quantum graphs, preprint (arXiv:0707.3710).

[36] A. Messiah, Quantum Mechanics, vol. I, Wiley, New York, 1961.

[37] K. A. Milton, The Casimir Effect: Physical Manifestations of Zero-Point Energy, World Scientific, Singapore, 2001.

[38] G. Plunien, B. Müller, and W. Greiner, The Casimir effect, Phys. Reps. 143 (1986) 87-193. 
[39] A. Romeo and A. A. Saharian, Casimir effect for scalar fields under Robin boundary conditions on plates, J. Phys. A 35 (2002) 1297-1320.

[40] J.-P. Roth, Le spectre du laplacien sur un graphe, in Théorie du Potentiel (G. Mokobodzki and D. Pinchon, eds.), Lec. Notes Math., vol. 1096, Berlin, Springer, 1984, pp. 521-539.

[41] M. Sieber, H. Primack, U. Smilansky, I. Ussishkin, and H. Schanz, Semiclassical quantization of billiards with mixed boundary conditions, J. Phys. A 28 (1995) 5041-5078.

[42] M. Sieber, U. Smilansky, S. C. Creagh, and R. G. Littlejohn, Non-generic spectral statistics in the quantized stadium billiard, J. Phys. A 26 (1993) 6217-6230.

[43] J. H. Wilson, J H 2007 Vacuum Energy in Quantum Graphs, Undergraduate Research Fellow thesis, Texas A\&M University, 2007 (http://handle.tamu.edu/1969.1/5682).

Departments of Mathematics and Physics, Texas A\&M University, College Station, TX, 77843-3368, USA

E-mail address: fulling@math.tamu.edu

Departments of Mathematics and Physics, Texas A\&M University, College Station, TX, 77843-3368, USA

Current address: Department of Physics, University of Maryland, College Park, MD, 207424111, USA

E-mail address: jwilson.thequark@gmail.com 